\title{
Optical variability patterns of radio-quiet and radio-loud quasars
}

\author{
P. Marziani ${ }^{1}$, E. Bon ${ }^{2}$, A. Grieco ${ }^{3}$, N. Bon ${ }^{2}$, D. Dultzin ${ }^{4}$, \\ A. Del Olmo ${ }^{5}$ and M. D'Onofrio ${ }^{6}$ \\ ${ }^{1}$ INAF, Osservatorio Astronomico di Padova, Italy \\ email: paola.marziani@oapd.inaf.it \\ ${ }^{2}$ Belgrade Observatory, Serbia \\ ${ }^{3}$ Via Carpane 78, Vigonza, Italy \\ ${ }^{4}$ Instituto de Astronomía, UNAM, Mexico \\ ${ }^{5}$ IAA (CSIC), Granada, Spain \\ ${ }^{6}$ Università di Padova, Italy
}

\begin{abstract}
We analyzed the light curves (LCs) of several radio-quiet and radio-loud quasars belonging to the same parameter space volume in the 4D Eigenvector 1 (4DE1) quasar sequence, using data from the Catalina Real-time Transient Survey (CRTS). We report preliminary results on detected variability pattern, and discuss possible cases of periodic variability.
\end{abstract}

Keywords. quasars: general, black hole physics, accretion, accretion disks, galaxies: jets

\section{Introduction}

The origin of the radio-quiet/radio-loud (RQ/RL) dichotomy is still not understood. In the present contribution, the optical variability patterns of RQ and RL low- $z$ ( $\lesssim$ 0.6) quasars are compared in the region of RL source maximum occupation along the eigenvector 1 sequence of Sulentic et al. (2000) in the plane FWHM of broad H $\beta$ vs. FeII prominence $\left(R_{\mathrm{FeII}}=I(\right.$ FeII $\left.\lambda 4570) / I(\mathrm{H} \beta)\right)$. Periodical variability may ultimately indicate the presence of a sub-parsec supermassive binary black hole (SMBBH). As a preliminary test to this hypothesis, we analyze whether RL and RQ quasars show systematic differences in variability measures, and identify some apparent cases of periodic variability.

\section{Quasar V-band variability patterns from the CRTS}

RL quasars are not distributed uniformly along the eigenvector 1 sequence, but are predominantly found in the area B1 with $R_{\mathrm{FeII}} \lesssim 0.5$ and $4000 \mathrm{~km} \mathrm{~s}^{-1} \lesssim \mathrm{FWHM}(\mathrm{H} \beta)$ $\lesssim 8000 \mathrm{~km} \mathrm{~s}^{-1}$ where also RQ quasars are found with similar accretion parameters $\left(\log L \sim 45.5\left[\mathrm{erg} \mathrm{s}^{-1}\right], \log M_{\mathrm{BH}} \sim 8.5, L / L_{\mathrm{Edd}} \sim 0.1\right)$. The CRTS (Drake et al. 2009) provided V-band magnitudes at 200 - 400 epochs for our sample of 77 B1 quasars (31 RL), over a time span of $\sim 3000 \mathrm{~d}$. The data allow for the computation of variability statistics as well as of the auto-correlation (ACF) and structure function (SF) of individual sources.

A two-sample comparison between RL and RQ sources in spectral bin B1 shows that (1) the excess variance distributions are not statistically different, according to KolmogorovSmirnov tests; (2) the rest-frame ACF 0 peak half-width (ACF-HW) distributions are marginally different (at slightly less than $2 \sigma$ confidence level; at $2 \sigma$ if the ACF-HW is normalized by the black hole mass). Two extreme cases are represented by III Zw 2 that shows a narrow ACF and is associated with no variability, and by PKS 2128-12 that shows 


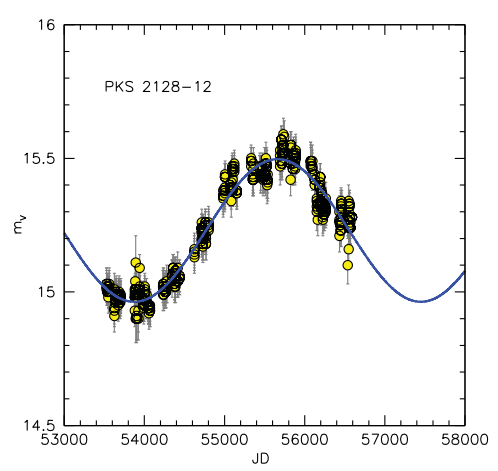

Figure 1. $V$-band light curve for PKS 2128-12 from CRST data. The blue curve represents a sine fit that corresponds to a period $P \approx 3576 \mathrm{~d}$. The excellent fit by a periodic function may indicate (but is absolutely not yet a proof of) periodic behavior.

a high degree of correlation between distant observations. Large ACF-HW values ( $\gtrsim 500$ d) are anyway possible for RL and RQ alike, which may indicate a stochastic process with a "red" frequency spectrum, or relatively long time-scale deterministic physical processes for both RQ and RL sources. In addition, Kolmogorov-Smirnov tests show that (3) the SF slopes are also not statistically different. The times and amplitudes of the SF maximum are slightly larger for RL than for RQ sources, although the two-sample difference is again not statistically significant. Nonetheless, RQ sources more often show an irregular multipeaked SF than RL ones. A larger sample of sources in the same domain the eigenvector 1 sequence is needed to confirm the results hinting at systematic RL/RQ differences in optical variability.

The inspection of several LCs suggests periodical behavior. The most impressive case in our sample is PKS 2128-12 (Fig. 1), with a light curve similar to the one of PKS 1302-102 (Graham et al. 2015), and of the compact-steep spectrum radio source 3C 57 (Sulentic et al. 2015). The Lomb-Scargle periodograms indicate that a large fraction of sources exhibit periodicity to some extent. However, quasars apparently show an optical LC consistent with damped random walks (e.g., MacLeod et al. 2012) where the stochastic variability amplitude grows larger at lower frequencies, thus creating the illusion of periodic curves (Vaughan et al. 2016). For instance, the peak corresponding to a period $P \approx 3600 \mathrm{~d}$ for PKS 2128-12 is highly significant against conventional Lomb-Scargle significance tests (associated with measurement errors) but not against red noise.

The CRTS is now covering the domain of deterministic processes dynamical timescales that can give rise to a periodic signal in luminous quasars. However, longer time coverages are needed to confirm periodicity in the candidates we identify, as for NGC 4151 and NGC 5548 (Bon et al. 2012, 2016). In both cases, periodicity was also detected in the radial velocity curves measured from the $\mathrm{H} \beta$ and $\mathrm{H} \alpha$ Balmer lines. Additional spectroscopic monitoring is needed for the CRTS candidates as well to track periodic behavior that may ultimately provide evidence in favor of sub-pc SMBBHs.

\section{References}

Bon, E., Jovanović, P., Marziani, P., et al. 2012, ApJ, 759, 118

Bon, E., Zucker, S., Netzer, H., et al. 2016, ApJS, 225, 29

Drake, A. J., Djorgovski, S. G., Mahabal, A., et al. 2009, ApJ, 696, 870

Graham, M. J., Djorgovski, S. G., Stern, D., et al. 2015, Nature, 518, 74

MacLeod, C. L., Ivezić, Ž., Sesar, B., et al. 2012, ApJ, 753, 106

Sulentic, J. W., Martínez-Carballo, M. A., Marziani, P., et al. 2015, MNRAS, 450, 1916

Sulentic, J. W., Zwitter, T., Marziani, P., \& Dultzin-Hacyan, D. 2000, ApJL, 536, L5

Vaughan, S., Uttley, P., Markowitz, A. G., et al. 2016, MNRAS, 461, 3145 